\title{
Further Results for Starlike Functions Related with Booth Lemniscate
}

\author{
Rahim Kargar ${ }^{1} \cdot$ Ali Ebadian $^{2} \cdot$ Lucyna Trojnar-Spelina $^{3}$ (D)
}

Received: 13 February 2018/Accepted: 1 June 2018/Published online: 18 June 2018

(C) The Author(s) 2018

\begin{abstract}
In this paper we investigate an interesting subclass $\mathcal{B S}(\alpha)(0 \leq \alpha<1)$ of starlike functions in the unit disc $\Delta$. The class $\mathcal{B S}(\alpha)$ was introduced by Kargar et al. (Anal Math Phys, 2017. https://doi.org/10.1007/s13324-017-0187-3) which is strongly related to the Booth lemniscate. Some geometric properties of this class of analytic functions, including radius of starlikeness of order $\gamma(0 \leq \gamma<1)$, the image of $f(\{z:|z|<r\})$ when $f \in \mathcal{B S}(\alpha)$, an special example and estimate of bounds for $\operatorname{Re}\{f(z) / z\}$, are studied.
\end{abstract}

Keywords Booth lemniscate $\cdot$ Radius of starlikeness $\cdot$ Starlike function $\cdot$ Convex function $\cdot$ Subordination

Mathematics Subject Classification 30C45

\section{Introduction}

Let $\mathcal{H}$ denote the class of analytic functions in the open unit disc $\Delta=\{z \in \mathbb{C}:|z|<1\}$ on the complex plane $\mathbb{C}$. Also let $\mathcal{A}$ denote the subclass of $\mathcal{H}$ including of functions normalized by $f(0)=f^{\prime}(0)-1=0$. The subclass of $\mathcal{A}$ that consists of all univalent functions $f(z)$ in $\Delta$ is denoted by $\mathcal{S}$. We denote by $\mathfrak{B}$ the class of functions $w(z)$ analytic in $\Delta$ with $w(0)=0$ and $|w(z)|<1,(z \in \Delta)$. For two analytic and normalized functions $f$ and $g$, we say that $f$ is subordinate to $g$, written $f \prec g$ in $\Delta$, if there exists a function $w \in \mathfrak{B}$ such that $f(z)=g(w(z))$ for all $z \in \Delta$. In special case, if the function $g$ is univalent in $\Delta$, then

$f(z) \prec g(z) \Leftrightarrow(f(0)=g(0)$ and $f(\Delta) \subset g(\Delta))$.

Lucyna Trojnar-Spelina

lspelina@prz.edu.pl

Rahim Kargar

rkargar1983@gmail.com

Ali Ebadian

aebadian@pnu.ac.ir

1 Young Researchers and Elite Club, Ardabil Branch, Islamic Azad University, Ardabil, Iran

2 Department of Mathematics, Faculty of Science, Urmia University, Urmia, Iran

3 Department of Discrete Mathematics, Faculty of Mathematics and Applied Physics, Rzeszów University of Technology, Rzeszów, Poland
It is easy to see that for any complex numbers $\lambda \neq 0$ and $\mu$, we have:

$f(z) \prec g(z) \Rightarrow \lambda f(z)+\mu \prec \lambda g(z)+\mu$.

The set of all functions $f \in \mathcal{A}$ that are starlike univalent in $\Delta$ will be denoted by $\mathcal{S}^{*}$ and the set of all functions $f \in \mathcal{A}$ that are convex univalent in $\Delta$ will be denoted by $\mathcal{K}$. Robertson (see Robertson 1985) introduced and studied the class $\mathcal{S}^{*}(\gamma)$ of starlike functions of order $\gamma \leq 1$ as follows

$\mathcal{S}^{*}(\gamma):=\left\{f \in \mathcal{A}: \quad \operatorname{Re}\left\{\frac{z f^{\prime}(z)}{f(z)}\right\}>\gamma, z \in \Delta\right\}$.

We note that if $\gamma \in[0,1)$, then a function in $\mathcal{S}^{*}(\gamma)$ is univalent. Also we say that $f \in \mathcal{K}(\gamma)$ (the class of convex functions of order $\gamma$ ) if and only if $z f^{\prime}(z) \in \mathcal{S}^{*}(\gamma)$. In particular we put $\mathcal{S}^{*}(0) \equiv \mathcal{S}^{*}$ and $\mathcal{K}(0) \equiv \mathcal{K}$.

Recently, Kargar et al. (2017) introduced and studied a class functions related to the Booth lemniscate as follows.

Definition 1.1 (see Kargar et al. 2017) The function $f \in \mathcal{A}$ belongs to the class $\mathcal{B S}(\alpha), 0 \leq \alpha<1$, if it satisfies the condition

$$
\left(\frac{z f^{\prime}(z)}{f(z)}-1\right) \prec \frac{z}{1-\alpha z^{2}} \quad(z \in \Delta) .
$$

Recall that (Piejko and Sokół 2013), a one-parameter family of functions given by 
$F_{\alpha}(z):=\frac{z}{1-\alpha z^{2}}=\sum_{n=1}^{\infty} \alpha^{n-1} z^{2 n-1} \quad(z \in \Delta, 0 \leq \alpha \leq 1)$.

are starlike univalent when $0 \leq \alpha \leq 1$ and are convex for $0 \leq \alpha \leq 3-2 \sqrt{2} \approx 0.1715$. We have also $F_{\alpha}(\Delta)=D(\alpha)$, where

$D(\alpha)=\left\{x+i y \in \mathbb{C}:\left(x^{2}+y^{2}\right)^{2}-\frac{x^{2}}{(1-\alpha)^{2}}-\frac{y^{2}}{(1+\alpha)^{2}}<0,(0 \leq \alpha<1)\right\}$

and

$$
\begin{aligned}
D(1)= & \{x+i y \in \mathbb{C}:(\forall t \in(-\infty,-i / 2] \cup[i / 2, \infty)) \\
& {[x+i y \neq i t]\} . }
\end{aligned}
$$

It is clear that the curve

$$
\left(x^{2}+y^{2}\right)^{2}-\frac{x^{2}}{(1-\alpha)^{2}}-\frac{y^{2}}{(1+\alpha)^{2}}=0 \quad(x, y) \neq(0,0) \text {, }
$$

is the Booth lemniscate of elliptic type (see Fig. 1, for $\alpha=1 / 3$ ). For more details, see Kargar et al. (2017).

Lemma 1.1 (see Kargar et al. 2017) Let $F_{\alpha}(z)$ be given by (1.3). Then for $0 \leq \alpha<1$, we have

$\frac{1}{\alpha-1}<\operatorname{Re}\left\{F_{\alpha}(z)\right\}<\frac{1}{1-\alpha} \quad(z \in \Delta)$.

Therefore by definition of subordination and by Lemma $1.1, f \in \mathcal{A}$ belongs to the class $\mathcal{B S}(\alpha)$, if it satisfies the condition

$\frac{\alpha}{\alpha-1}<\operatorname{Re}\left\{\frac{z f^{\prime}(z)}{f(z)}\right\}<\frac{2-\alpha}{1-\alpha} \quad(z \in \Delta)$.

The following lemma will be useful.

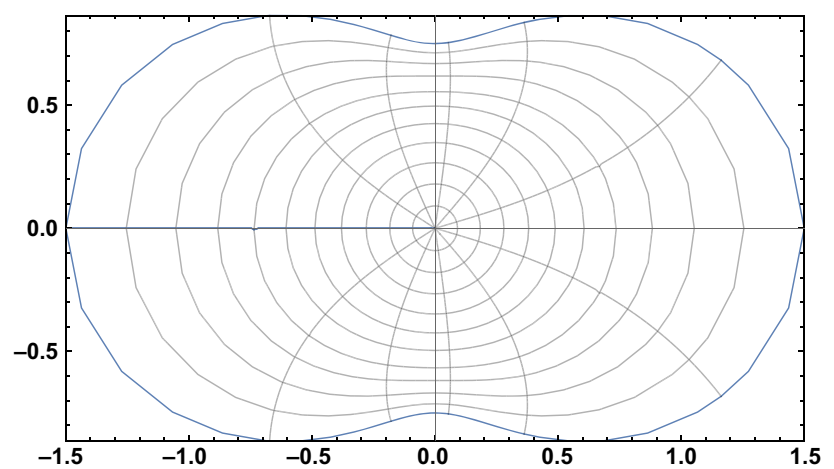

Fig. $1\left(x^{2}+y^{2}\right)^{2}-9 x^{2} / 4-9 y^{2} / 16=0$
Lemma 1.2 (see Ruscheweyh and Stankiewicz 1985) Let $F, G \in \mathcal{H}$ be any convex univalent functions in $\Delta$. If $f \prec F$ and $g \prec G$, then

$f * g \prec F * G$ in $\Delta$.

In this work, some geometric properties of the class $\mathcal{B S}(\alpha)$ are investigated.

\section{Main Results}

We start with the following lemma that gives the structural formula for the function of the considered class.

Lemma 2.1 The function $f \in \mathcal{A}$ belongs to the class $\mathcal{B S}(\alpha), 0 \leq \alpha<1$, if and only if there exists an analytic function $q, q(0)=0$ and $q \prec F_{\alpha}$ such that

$f(z)=z \exp \left(\int_{0}^{z} \frac{q(t)}{t} \mathrm{~d} t\right)$.

The proof is easy. Putting $q=F_{\alpha}$ in Lemma 2.1 we obtain the function

$\tilde{f}(z)=z\left(\frac{1+z \sqrt{\alpha}}{1-z \sqrt{\alpha}}\right)^{\frac{1}{2 \sqrt{\alpha}}}$

which is extremal function for several problems in the class $\mathcal{B S}(\alpha)$. Moreover, we consider

$$
F(z):=\frac{\tilde{f}(z)}{z}=\left(\frac{1+z \sqrt{\alpha}}{1-z \sqrt{\alpha}}\right)^{\frac{1}{2 \sqrt{\alpha}}}=1+z+\frac{1}{2} z^{2}+\frac{1}{3}\left(\alpha+\frac{1}{2}\right) z^{3} \cdots .
$$

From (1.7) we conclude that $f \in \mathcal{B S}(\alpha)$ is starlike of order $\frac{\alpha}{\alpha-1}<0$, hence $f$ may not be univalent in $\Delta$. It may therefore be interesting to consider a problem to find the radius of starlikeness of order $\gamma, \gamma \in[0,1)$ (hence univalence) of the class $\mathcal{B S}(\alpha)$, i.e. the largest radius $r_{s}(\alpha, \gamma)$ such that each function $f \in \mathcal{B S}(\alpha)$ is starlike of order $\gamma$ in the disc $|z|<r_{s}(\alpha, \gamma)$. For this purpose we recall the following property of the class $\mathfrak{B}$.

Lemma 2.2 (Schwarz lemma) (see Duren 1983) Let $w$ be analytic in the unit disc $\Delta$, with $w(0)=0$ and $|w(z)|<1$ in $\Delta$. Then $\left|w^{\prime}(0)\right| \leq 1$ and $|w(z)| \leq|z|$ in $\Delta$. Strict inequality holds in both estimates unless $w$ is a rotation of the disc: $w(z)=e^{i \theta} z$.

Theorem 2.1 Let $\alpha \in(0,1)$ and $\gamma \in[0,1)$ be given numbers. If $f \in \mathcal{B S}(\alpha)$, then $f$ is starlike of order $\gamma$ in the disc $|z|<r_{s}(\alpha, \gamma)=\frac{\sqrt{1+4 \alpha(1-\gamma)}-1}{2 \alpha(1-\gamma)}$. The result is sharp. 
Proof Let $f \in \mathcal{B S}(\alpha), \alpha \in(0,1)$. Then through (1.2) we have $\left(\frac{z f^{\prime}(z)}{f(z)}-1\right) \prec \frac{z}{1-\alpha z^{2}}$ so there exists $w \in \mathfrak{B}$ such that $\operatorname{Re}\left\{\frac{z f^{\prime}(z)}{f(z)}\right\}=\operatorname{Re}\left\{\frac{1+w(z)-\alpha w^{2}(z)}{1-\alpha w^{2}(z)}\right\}$

for all $z \in \Delta$. Applying the Schwarz lemma we obtain

$$
\begin{aligned}
\operatorname{Re}\left\{\frac{z f^{\prime}(z)}{f(z)}\right\} & =\operatorname{Re}\left\{1+\frac{w(z)}{1-\alpha w^{2}(z)}\right\}=1+\operatorname{Re}\left\{\frac{w(z)}{1-\alpha w^{2}(z)}\right\} \\
& \geq 1-\frac{|w(z)|}{1-\alpha|w(z)|^{2}} \geq 1-\frac{|z|}{1-\alpha|z|^{2}}=1-\frac{r}{1-\alpha r^{2}},
\end{aligned}
$$

where $r=|z|<1$. Let us consider a function $h(r)=1-$ $\frac{r}{1-\alpha r^{2}}, r \in(0,1)$. Note that $h^{\prime}(r)=-\frac{1+\alpha r^{2}}{\left(1-\alpha r^{2}\right)^{2}}<0$ for all $r \in$ $[0,1)$ hence $h$ is a strictly decreasing function and it decreases from 1 to $\frac{\alpha}{\alpha-1}<0$. Therefore the equation $h(r)=$ $\gamma$ has for given $\alpha$ and $\gamma$ the smallest positive root $r_{s}(\alpha, \gamma)$ in $(0,1)$. Therefore $f$ is starlike of order $\gamma$ in $|z|<r \leq r_{s}(\alpha, \gamma)$. Note that for the function $\tilde{f}$ given in (2.2) we obtain

$\operatorname{Re} \frac{z \tilde{f}^{\prime}(z)}{\tilde{f}(z)}=\operatorname{Re}\left\{1+\frac{z}{1-\alpha z^{2}}\right\}=: A(z)$

and $A\left(-r_{s}(\alpha, \gamma)\right)=\gamma$.

Putting $\gamma=0$ in Theorem 2.1, we obtain.

Corollary 2.1 Let $\alpha \in(0,1)$. If $f \in \mathcal{B S}(\alpha)$ then $f$ is starlike univalent in the disc $|z|<r_{s}(\alpha)=\frac{\sqrt{1+4 \alpha}-1}{2 \alpha}$. The result is sharp.

Remark 2.1 Note that $\lim _{\alpha \longrightarrow 0^{+}} r_{s}(\alpha)=$ $\lim _{\alpha \longrightarrow 0^{+}} \frac{2}{\sqrt{1+4 \alpha}+1}=1$. Moreover, it is worth mentioning that $\lim _{\alpha \longrightarrow 1^{-}} r_{s}(\alpha)=\frac{\sqrt{5}-1}{2}=0,618 \ldots$, i.e. this limit is a reciprocal of the golden ratio $\frac{\sqrt{5}+1}{2}$.

Now we consider the following question:

For a given number $r \in(0,1]$ find $\alpha(r)$ such that for each function $f \in \mathcal{B S}(\alpha(r))$ the image $f(\{z:|z|<r\})$ is a starlike domain.

Theorem 2.2 Let $r \in(0,1]$ be the given number. If $0 \leq \alpha<\frac{1-r}{r^{2}}$, then each function $f \in \mathcal{B S}(\alpha)$ maps a disc $|z|<r$ onto a starlike domain.

Proof After using the same argument as in the proof of Theorem 2.1 we conclude that $f \in \mathcal{B S}(\alpha)$ satisfies the equality

$\operatorname{Re}\left\{\frac{z f^{\prime}(z)}{f(z)}\right\}=\operatorname{Re}\left\{\frac{1+w(z)-\alpha w^{2}(z)}{1-\alpha w^{2}(z)}\right\}$

for all $z \in \Delta$ with some $w \in \mathfrak{B}$. Then we have by Schwarz's lemma that
$\operatorname{Re}\left\{\frac{z f^{\prime}(z)}{f(z)}\right\} \geq 1-\frac{|w(z)|}{1-\alpha|w(z)|^{2}} \geq 1-\frac{|z|}{1-\alpha|z|^{2}}$.

Consequently, for $|z|<r$, we obtain $\operatorname{Re}\left\{\frac{z f^{\prime}(z)}{f(z)}\right\}>1-\frac{r}{1-\alpha r^{2}}$. Let us note that a function $g(\alpha)=1-\frac{{ }_{r}}{1-\alpha r^{2}}, \alpha \in[0,1)$, has positive values for $0 \leq \alpha<\frac{1-r}{r^{2}}$. Therefore the image of the disc $|z|<r$ is a starlike domain.

Theorem 2.3 Let $n \geq 2$ be integer. If one of the following conditions holds

$$
\frac{1}{\alpha+n(1-\alpha)}<|c|<1
$$

(ii) $n>\frac{3-\alpha}{1-\alpha}$ and $\frac{1}{\alpha-2+n(1-\alpha)}<|c|<1$,

(iii) $n \geq \frac{2-\alpha}{1-\alpha}$ and $|c|>1$,

(iv) $n<\frac{2-\alpha}{1-\alpha}$ and $1<|c|<\frac{1}{2-\alpha+n(\alpha-1)}$,

then the function $g_{n}(z)=z+c z^{n}$ does not belong to the class $\mathcal{B S}(\alpha)$.

Proof Let us put $G(z)=\frac{z g_{n}^{\prime}(z)}{g_{n}(z)}-1=\frac{1+c n z^{n-1}}{1+c z^{n-1}}-1$. To prove our assertion it suffices to show that the function $G$ is not subordinate to $F_{\alpha}$ or equivalently, because of the univalence of the dominant function $F_{\alpha}$, that the set $G(\Delta)$ is not included in $F_{\alpha}(\Delta)=D(\alpha)$. Upon performing simple calculation we find that the set $G(\Delta)$ is the disc with the diameter from the point $x_{1}=\frac{|c|(n-1)}{|c|-1}$ to the point $x_{2}=$ $\frac{|c|(n-1)}{|c|+1}$. The set $D(\alpha)$ is bounded by the curve

$$
\left(x^{2}+y^{2}\right)^{2}-\frac{x^{2}}{(1-\alpha)^{2}}-\frac{y^{2}}{(1+\alpha)^{2}}=0, \quad(x, y) \neq(0,0) \text {. }
$$

We have $\min _{|z|=1} \operatorname{Re}\left\{F_{\alpha}(z)\right\}=F_{\alpha}(-1)=\frac{1}{\alpha-1} \quad$ and $\max _{|z|=1} \operatorname{Re}\left\{F_{\alpha}(z)\right\}=F_{\alpha}(1)=\frac{1}{1-\alpha}$. If one of the conditions $(i)-(i v)$ is satisfied then $\min \left\{x_{1}, x_{2}\right\}<\frac{1}{\alpha-1}$ or $\max \left\{x_{1}, x_{2}\right\}>\frac{1}{1-\alpha}$, and then $G(\Delta)$ is not included in $D(\alpha)$. The proof of theorem is completed.

Recently, one of the interesting problems for mathematician is to find bounds for $\operatorname{Re}\{f(z) / z\}$ (see Kargar et al. 2016; Sim and Kwon 2013). In the sequel, we obtain lower and upper bounds for $\operatorname{Re}\{f(z) / z\}$. We first get the following result for the function $F(z)$ given by (2.3).

Theorem 2.4 The function $F(z)$ of the form (2.3) is convex univalent in $\Delta$.

Proof A simple calculation gives us

$$
1+\frac{z F^{\prime \prime}(z)}{F^{\prime}(z)}=1+\left(\frac{1}{2 \sqrt{\alpha}}-1\right)\left(\frac{2 \sqrt{\alpha} z}{1-\alpha z^{2}}\right)+\frac{2 \sqrt{\alpha} z}{1-\sqrt{\alpha} z} .
$$

It is sufficient to show that (2.4) has positive real part in the unit disc. From Lemma 1.1 we obtain 


$$
\begin{aligned}
& \operatorname{Re}\left\{1+\frac{z F^{\prime \prime}(z)}{F^{\prime}(z)}\right\}=\operatorname{Re}\left\{1+\left(\frac{1}{2 \sqrt{\alpha}}-1\right)\left(\frac{2 \sqrt{\alpha} z}{1-\alpha z^{2}}\right)+\frac{2 \sqrt{\alpha} z}{1-\sqrt{\alpha} z}\right\} \\
= & 1+2 \sqrt{\alpha}\left(\frac{1}{2 \sqrt{\alpha}}-1\right) \operatorname{Re}\left\{\frac{z}{1-\alpha z^{2}}\right\}+2 \sqrt{\alpha} \operatorname{Re}\left\{\frac{z}{1-\sqrt{\alpha} z}\right\} \\
> & 1+(1-2 \sqrt{\alpha})\left(\frac{1}{\alpha-1}\right)-\frac{2 \sqrt{\alpha}}{1+\sqrt{\alpha}}=: K(\alpha) \quad(0 \leq \alpha<1) .
\end{aligned}
$$

It is easily seen that $K^{\prime}(\alpha)=\frac{1}{(\alpha-1)^{2}}>0$. Thus $K(\alpha) \geq K(0)=0$, and hence $F(z)$ is convex univalent function.

In the proof of the next theorem we will use the following result concerning the convexity of the boundary of $D(\alpha)$.

Lemma 2.3 (see Piejko and Sokół 2013) Suppose that $F_{\alpha}$ is given by (1.3). If $0 \leq \alpha \leq 3-2 \sqrt{2} \approx 0.1715$, then the curve $F_{\alpha}\left(e^{i \varphi}\right), \varphi \in[0,2 \pi)$, is convex. If $\alpha \in(3-2 \sqrt{2}, 1)$, then the curve $F_{\alpha}\left(e^{i \varphi}\right), \varphi \in[0,2 \pi)$, is concave. Moreover, in both cases this curve is symmetric with respect to both axes.

Theorem 2.5 If a function $f$ belongs to the class $\mathcal{B S}(\alpha)$, $0 \leq \alpha \leq 3-2 \sqrt{2}$, then

$\frac{f(z)}{z} \prec F(z) \quad(z \in \Delta)$,

where $F(z)$ is given by (2.3).

Proof Let $0 \leq \alpha \leq 3-2 \sqrt{2}$ and let $f$ be in the class $\mathcal{B S}(\alpha)$. Then we have

$\phi(z):=\frac{z f^{\prime}(z)}{f(z)}-1 \prec F_{\alpha}(z) \quad(z \in \Delta)$,

where $F_{\alpha}$ is given by (1.3). It is well known that the normalized function

$l(z)=\log \frac{1}{1-z}=\sum_{n=1}^{\infty} \frac{z^{n}}{n} \quad(z \in \Delta)$,

belongs to the class $\mathcal{K}$ and for $f \in \mathcal{A}$ we get

$\phi(z) * l(z)=\int_{0}^{z} \frac{\phi(t)}{t} \mathrm{~d} t \quad$ and $\quad F_{\alpha}(z) * l(z)=\int_{0}^{z} \frac{F_{\alpha}(t)}{t} \mathrm{~d} t$.

By Lemma 2.3 we deduce that the function $F_{\alpha}$ is convex.

Thus applying Lemma 1.2 in (2.6) we obtain

$\phi(z) * l(z) \prec F_{\alpha}(z) * l(z) \quad(z \in \Delta)$.

Now from (2.7) and (2.8), we can obtain

$\int_{0}^{z} \frac{\phi(t)}{t} \mathrm{~d} t \prec \int_{0}^{z} \frac{F_{\alpha}(t)}{t} \mathrm{~d} t \quad(z \in \Delta)$.
Thus

$\frac{f(z)}{z}=\exp \int_{0}^{z} \frac{\phi(t)}{t} \mathrm{~d} t \prec \int_{0}^{z} \frac{F_{\alpha}(t)}{t} \mathrm{~d} t=\frac{\tilde{f}(z)}{z}$.

This completes the proof of theorem.

Here by combining Theorem 2.4, Theorem 2.5 and (1.1), we get:

Theorem 2.6 Let $f \in \mathcal{B S}(\alpha), \quad 0 \leq \alpha \leq 3-2 \sqrt{2}$ and $|z|=r<1$. Then

$$
\left(\frac{1-r \sqrt{\alpha}}{1+\sqrt{\alpha}}\right)^{\frac{1}{2 \sqrt{\alpha}}} \leq \operatorname{Re}\left(\frac{f(z)}{z}\right) \leq\left(\frac{1+r \sqrt{\alpha}}{1-r \sqrt{\alpha}}\right)^{\frac{1}{2 \sqrt{\alpha}}} \quad(z \in \Delta) \text {. }
$$

The result is sharp.

Proof By the subordination principle, we have:

$f(z) \prec g(z) \Rightarrow f(|z|<r) \subset g(|z|<r) \quad(0 \leq r<1)$.

From Theorem 2.4, since $F(z)$ is convex univalent in $\Delta$, and it is real for real $z$, thus it maps the disc $|z|=r<1$ onto a convex set symmetric which respect to the real axis laying between $F(-r)-1$ and $F(r)-1$. Now the assertion is obtained from Theorem 2.5.

Open Access This article is distributed under the terms of the Creative Commons Attribution 4.0 International License (http://creative commons.org/licenses/by/4.0/), which permits unrestricted use, distribution, and reproduction in any medium, provided you give appropriate credit to the original author(s) and the source, provide a link to the Creative Commons license, and indicate if changes were made.

\section{References}

Duren PL (1983) Univalent functions. Springer, Berlin

Kargar R, Ebadian A, Sokół J (2016) On subordination of some analytic functions. Sib Math J 57:599-604

Kargar R, Ebadian A, Sokół J (2017) On Booth lemniscate and starlike functions. Anal Math Phys. https://doi.org/10.1007/ s13324-017-0187-3

Piejko K, Sokół J (2013) Hadamard product of analytic functions and some special regions and curves. J Inequal Appl 2013:420

Robertson MS (1985) Certain classes of starlike functions. Mich Math J 32:135-140

Ruscheweyh St, Stankiewicz J (1985) Subordination under convex univalent function. Bull Pol Acad Sci Math 33:499-502

Sim YJ, Kwon OS (2013) Notes on analytic functions with a bounded positive real part. J Inequal Appl 2013:370. https://doi.org/10. 1186/1029-242X-2013-370 